\title{
Investigating Service Discovery, Management and Network Support for Next Generation Object Oriented Services
}

\author{
Bilhanan Silverajan, Joona Hartman, and Jani Laaksonen \\ Dept. of Information Technology, Tampere University of Technology, P.O. Box 553, FIN-33101 \\ Tampere, Finland
}

\{bilhanan|hartmanj|jjlaakso\}@cs.tut.fi

\begin{abstract}
The network computing industry has eagerly embraced technologies, welcoming an ever-increasing variety of new service discovery protocols and object architectures. With this abundance now offered across a wide collection of environments, technologies that offer standardized interfaces for the discovery process, while supporting communication for several different types of service access technologies, will provide the greatest achievable interoperability and resilience in the long-term. In this paper, we introduce a distributed architecture based on using directory services to significantly reduce the complexity of managing the information and services required to support next-generation networked applications, by providing automatic service discovery and a single coherent model for representing the data managed by supporting services. Standards-based solutions are used, and a prototype implementation of the CORBA Naming Service that has been designed to illustrate how the architecture incorporates distributed object models, directory services and multicast-based dynamic service discovery is presented.
\end{abstract}

Keywords: Distributed Object Architectures, Next-Generation Applications, Directory Services, Service Discovery

The original version of this chapter was revised: The copyright line was incorrect. This has been corrected. The Erratum to this chapter is available at DOI: 10.1007/978-0-387-35584-9_19 


\section{INTRODUCTION}

Recent years have witnessed the network and distributed computing industry embracing various technologies, media and content as a direct consequence of converging technical and business interests of telecommunications networks and Internet service providers. In the long run, this will likely lead to integration of all types of communications: a bewildering array of devices such as mobiles, fixed phones, PDAs, embedded devices, workstations and PCs will all be used to provide seamless communications between users and services. This has led to significant investment by many service and content providers into developing and furthering network and protocol-oriented technologies, for it is widely accepted that such a convergence will likely result in a structurally stable core packet-switched public network such as the Internet, having many edges acting as private gateways to proprietary and subscriber-based intranets offering a multitude of services using several different kinds of technology. These private networks may be both circuit and packet switched networks and will comprise subscriber-based IP connections, enterprise nodes and services, fixed and mobile telephone connections, intelligent network services and so on.

In this paper, we introduce a distributed architecture based on using directory services to significantly reduce the complexity of managing the information and services required to support next-generation networked applications, by providing automatic service discovery and a single coherent model for representing the data managed by supporting services. The architecture promises to be scalable and flexible enough to address basic issues that will arise in distributed computing as well as user, device and application mobility in the future. Section 2 discusses the type of network connectivity and support necessary to cope with the rising demand of mobile networked users and applications. Section 3 then sets out the aims and objectives of the proposed architecture while Section 4 provides an overview and a discussion of the proposed architecture, as well as describing a prototype that was implemented. Section 5 presents some performance measurements that were made, with the conclusions in Section 6.

\section{REQUIREMENTS FOR NETWORK SUPPORT}

Regardless of how services are utilized, two dominant scenarios are nevertheless heavily anticipated to influence and drive the development of next generation networks: User (and device) mobility and application mobility. 


\subsection{User mobility}

Network connectivity and support for user mobility is a necessary measure which is already being provisioned for in many organizations. The overall demand for mobility has indeed not shown any significant decrease, as evident by the tremendous popularity and ever-increasing adoption of low cost portable devices such as mobile computing devices, PDAs and phones for computing and networking needs. This trend will very likely be even more widespread, given that roaming agreements signed amongst mobile operators may not only include current demands for seamless GSM-based voice or data calls, but might encompass future GPRS and UMTS enabled service.

In supporting user mobility, user identities, profiles, and sessions will need to be preserved across network boundaries. Very often user mobility is associated with device mobility, in which the mobile device retains its identity when roaming. The user may thus have an associated device which moves with him but the user may remain unaware of the underlying networking issues such as whether the device assuming temporary network addresses on roaming networks or retaining its address during base station handovers.

However, some applications resident on the device may need an awareness of the current location to access local services such as printers and file servers and might thus have to perform service discovery to a certain extent. Furthermore, visiting devices may also enrich the network being visited by offering their own services to the various applications and users that are resident on that particular network. Access control mechanisms would thus need to be exerted by the network hosting the user to ascertain exactly which local services are visible and what levels of service utilization are permitted.

There are several technologies which exist today that inherently support user and device mobility. These include wireless LAN technologies such as $802.11 \mathrm{~b}$, Bluetooth technology for piconet and scatternet oriented ad-hoc networking, as well as movement across networks as specified by mobile IP.

\subsection{Application mobility}

Application mobility can occur independently of user and device mobility, in a sense that all or certain parts of an application may autonomously or semi-autonomously migrate across heterogenous network spaces. An application may also consist of numerous non-mobile components or objects which transparently reside in several different parts of a network, with each component offering well known services to the others. 
Network connectivity and support for application mobility on the other hand is foreseen in the medium to long-term future. It can be regarded as the next major wave in mobility after user and device mobility, which will have an important bearing on the underlying infrastructure.

Such an approach for application development is being undertaken in mobile code and agent technologies and frameworks, as well as distributed object-oriented architectures. Current examples include Java programs, automatic downloads of codecs by multimedia applications, Jini[1]-enabled programs, CORBA [2] applications and the forthcoming distributed applications of the Microsoft .NET framework [3]. Location awareness will be of lesser importance for migration when compared to computing resource availability of the present host and network.

Owing to the transparent, distributed and seemingly autonomous nature of such applications, it is rather unlikely they would enjoy as rapid and widespread an adoption across untrusted commercial networks as user and device mobility would. Rather, it is more probable that application mobility would garner more support and fill a demand for important niche services in enterprise level computing and service provisioning, within corporate intranets as well as private research networks of a more academic nature.

\subsection{Supporting requirements}

Such an environment of connectivity and mobility will have a significant impact on the way next-generation applications, both autonomous and interactive are implemented, discovered, managed and accessed. One can easily perceive that both user mobility and application mobility will become killer scenarios for existing networks and services. These will create an overwhelming strain in the way current networks and legacy applications manage, interact and serve the necessary information that needs to be delivered to the end-users and applications. In both cases, a more robust supporting infrastructure for handling next-generation applications needs to be developed in a standardized manner that must also prove scalable and adapt to accommodate the research and development challenges that will manifest themselves.

The overall design must possess some flexibility, as applications being built today are likely to function as part of bigger, integrated systems tomorrow. The architect of tomorrow's distributed systems will face largescale distributions both in terms of the number of users and connected systems running atop heterogenous networks. There may not be a revolutionary change in the way networks and services will be designed for supporting next-generation applications. In fact, all indications point to the development being evolutionary, with network architects and scientists 
examining, using and extending existing solutions and standards to support new applications and services as the need arises.

The huge potential of being able to harness an increased subscriber base of a converged market has led to several sets of standards continuously being introduced for communication protocols and architectures. In many ways, the objectives and target applications to be supported by these technologies can be remarkably similar. Even the technologies may mirror each other. However, in reality, getting applications and services supported by one set of standards to communicate with another pose interoperability challenges. This also leads to an unfortunate effect of leading to fragmented or duplicate solutions. As an example, IETF's Service Location Protocol [4], Sun Microsystems' Jini, Microsoft's UPnP [5] and wireless technologies promoted by IrDA and Bluetooth all implement their own service discovery mechanisms for potentially common types of services such as printing. Another notable example would include an object location service such as CORBA and RMI naming services.

Thus, a service designer targeting a product for multiple networking environments, object services or service discovery methods may be faced with the dilemma having to develop and managing several different kinds of applications, each supporting a specific protocol or object model but in all probability having duplicate information models.

Instead, trying to achieve the same goal by supporting protocol bridging and conversion methods for several service discovery methods and location services, with different front-ends using a common back-end applicationlevel information repository storing service records and attributes, would be a far less harrowing experience.

The challenge then becomes one of developing a unified yet scalable service information management system that stores and manages service data in a standardized and generic manner, but still provides the appropriate data to requesting application via a specific discovery method, using gateways and information mapping and translation.

\section{AIMS AND OBJECTIVES}

A prototype distributed environment that would enable devices, objectoriented client applications, and services to automatically search for a particular capability, and to request and establish interoperable sessions with other clients or servers was investigated to meet the following objectives:

- To provide applications, services and devices a standard method for describing and advertising their capabilities 
- To allow mobile users and applications the possibility to discover local services upon application start-up or movement within network space or administrative zones automatically or with as little static service discovery configuration as possible

- Interworking non-invasively or transparently with existing code and applications

- Ease of administration and maintenance by providing a single consistent model of available services that can scale, at the very minimum, to meet enterprise computing needs, thus reducing or eliminating the need for duplicity of information needed to support various access methods

- Integration into existing or commonly prevalent network resources, supporting components and infrastructure that may already be in place, but remaining extensible enough to meet future needs

- Well-known user and application access control and security mechanisms can be enforced, and existing authentication practices and encryption methods within the organization can be used.

\section{ARCHITECTURAL OVERVIEW}

Figure 1 illustrates a prototype implementation of a CORBA Naming Service [6] that has been designed to fulfill the objectives mentioned in section 3. CORBA has become increasingly important in distributed computing for the Internet as well as telecommunications owing to its language independence and separation of the object interface away from its implementation, and the increased use of distributed objects needing fundamental CORBA based object services such as the Naming Service cannot be ignored.

In this prototype, the back end service repository was implemented as a directory service using the Lightweight Directory Access Protocol (LDAP) [7]. This allowed the possibility to leverage the existing prevalence of using LDAP-based services and servers based on a well-known IETF-standard protocol already commonly deployed at the organizational level. Moreover much research is being done to make LDAP services secure, scalable, distributed and fault-tolerant. Clients would be able to retrieve information about services either natively through LDAP, or through a lightweight gateway/proxy that will implement the specific mappings necessary to support the particular type of service technology (in this case the CORBA Naming Service) the client would be using. As the figure shows, the model would be capable of supporting other kinds of front-ends as well. 


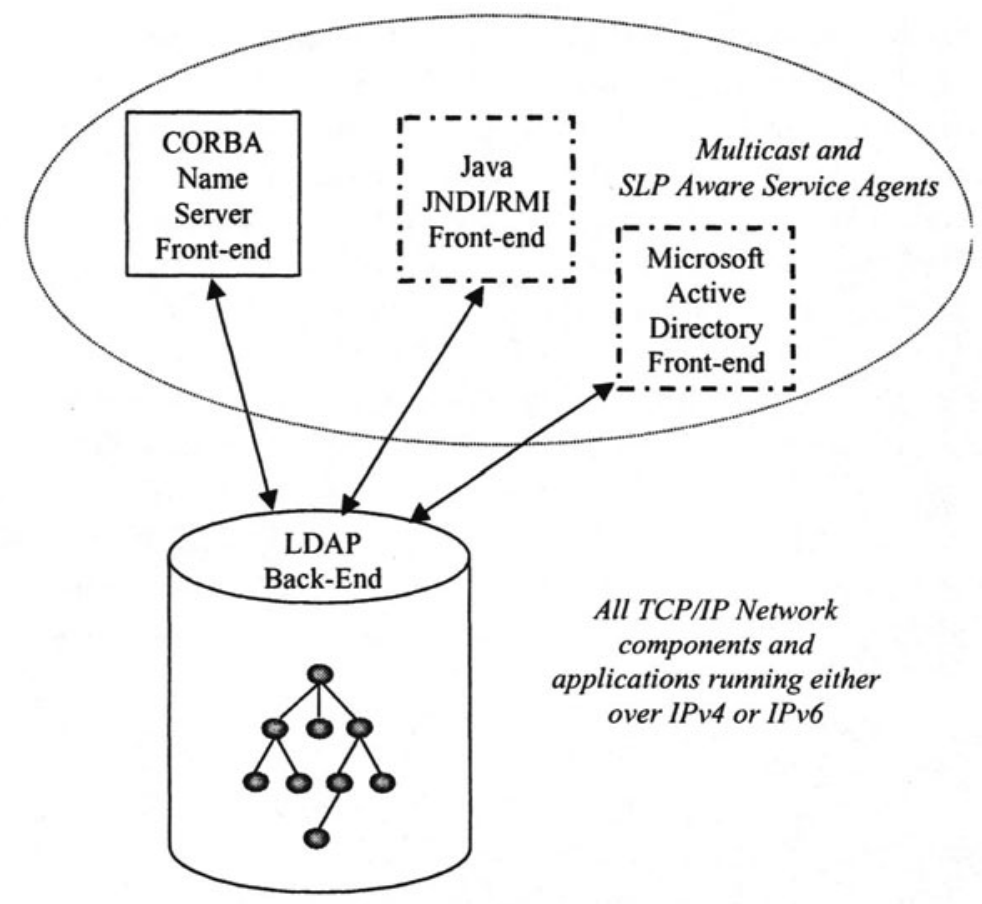

Figure 1. Prototype directory-based CORBA Naming Service

The discovery of the CORBA Naming Service by CORBA client and server objects was made possible using the Service Location Protocol (SLP) as a bootstrapping protocol for multicast-based for service discovery.

A brief description of SLP, LDAP and the CORBA Naming Service together with how they have been used or implemented are discussed in the following subsections.

\subsection{Automatic service discovery using SLP}

The Service Location Protocol (SLP) provides a flexible and scalable framework for providing hosts with access to information about the existence, location, and configuration of networked services. Traditionally, users have had to find services by knowing the name of a network host or its network address [4]. SLP eliminates the need for a user to know the name of a network host supporting a service. Rather, the user supplies the desired type of service and a set of attributes which describe the service. Based on that description, SLP resolves the network address of the service for the user.

SLP models client applications as User Agents (UAs), while services are advertised by Service Agents (SAs). Applications which would like to advertise their respective services use IETF standardized API functions to 
contact the SA running on the same host to register a Uniform Resource Location (URL) to be advertised, such as "service:ftp://ftp.company.com/". A service can moreover have attributes that describe the service (or the URL) in more detail. As an example, a web server would have its URL as "service:http://www.company.com" with attributes possibly listing the name, email address or a telephone number of the webmaster. SLP also allows services to be administratively grouped into scopes that could then be controlled for service provisioning to UAs.

Client programs (UAs) that would like to find services in a network also use standardized IETF function calls to find available services in a network and are capable of querying available service types, specific service type URLs as well as attributes associated with a specific URL. Directory Agents (DAs) are an optional third category of agents that SLP defines which allows for scalability by caching SA advertisements for direct interaction with UAs. Service discovery can either be statically configured or allowed to take advantage of multicasting or broadcasting features in a network for dynamic configuration.

The choice of using SLP as a primary service discovery protocol was influenced by the fact that it has been specified by the IETF, and hence did not need to be adapted to be used over TCP/IP. Moreover, it is generic and independent of any particular language and object architecture. SLP's simplicity combined with its feature set also enables it to interwork with various other service discovery methods and environments such as Jini using bridging [8] and the Apple Computer's next-generation AppleTalk [9]. Much standardization work is also in progress, such as efforts in combining SLP with LDAP [10], and extensions for SLP for use over IPv6 [11].

An open-source implementation of SLP, named OpenSLP [12] that implements SLP version 2, was primarily used in developing the SLP UA and SA components of the architecture. In the later stages of development and testing, the SLP system libraries of Sun's Solaris 8 were also used successfully. As only a minimum configuration was involved, no Directory Agents were used, and the architecture was developed to use dynamic discovery and communication between UAs and SAs using multicasting instead.

\subsection{Information storage and retrieval using LDAP}

LDAP was originally developed as a front end to X.500, the OSI directory service. X.500 defines the Directory Access Protocol (DAP) for clients to use when contacting directory servers. Currently at version 3, LDAP however has evolved to eventually provide most of the functionality of DAP at a much lower cost and defines a reasonably simple mechanism for 
Internet clients to query and manage an arbitrary database of hierarchical attribute/value pairs over a TCP/IP connection. LDAP has rapidly gaining significant Internet support, including the support of many companies, such as Novell, Sun, HP, IBM, SGI, AT\&T and Banyan, and is the focus of much standardization activity in the IETF.

The LDAP directory service model is based on entries. An entry is a collection of attributes that has a name, called a distinguished name (DN). The DN is used to refer to the entry unambiguously. Each of the entry's attributes has a type and one or more values. The types are typically mnemonic strings, like "cn" for common name, or "mail" for e-mail address. The values depend on what type of attribute it is. Directory entries are arranged in a hierarchical tree-like structure that reflects political, geographic, and/or organizational boundaries, representing people, organizational units, printers, documents, or just about anything else one can think of.

The information model stored in the LDAP directory backend of our prototype was loosely modeled upon the campus computer science department building, in both the physical and organizational sense. The top level root entry $(\mathrm{o}=\mathrm{CS}, \mathrm{c}=\mathrm{FI})$ represents the computer science building which was then subdivided into four floors representing the structure, personnel, laboratories, services and IP subnets resident on these floors. The basic idea in storing service and object information in the LDAP directory in this manner was not only to store the access method of the object or the URL of the service, but also to append the geological or organizational location information of the respective services and objects. Any LDAP browser could then be used in obtaining detailed information about services offered within particular subnet or subnets (such as printers), by inspecting the relevant attributes of the LDAP entries, such as their network addresses, physical location as well as different access methods (network printing, Bluetooth/IrDA connectivity, Appletalk, and so on). The OpenLDAP [13] project provided the necessary tools and libraries needed for this part of the architecture.

\subsection{Object location discovery using CORBA Naming Service}

The Naming Service was implemented in C++ with MICO [14], an opensource CORBA implementation which conforms to CORBA 2.3, using the DSI (Dynamic Skeleton Interface) to dynamically handle the object invocations. Apart from being designed specifically to aid in the development of gateways, the CORBA DSI functionality will allow the possibility to expand the interface of the CORBA naming service to possibly 
serve other kinds of naming services, since type-checking is done at runtime. Clients are free to use either the DII (Dynamic Invocation Interface) or the SII (Static Invocation Interface) to invoke the methods on the server object.

The CORBA objects are stored in the directory as stringified object references. LDAP offers a schema for representing CORBA Object References in an LDAP Directory [15], with each entry storing a textual description of the CORBA object as well as its IOR (Interoperable Object Reference). The IOR is the attribute with the single-most relevance in this case, as it is the primary means used by CORBA clients to locate and communicate with CORBA server objects in a network. Figure 2 displays the LDAP directory as seen by a freeware LDAP browser, Softerra LDAP Browser [16] running on Windows2000, after the Naming Service was launched and was used to register a simple CORBA Messaging server application, showing its various attributes, location as well as its DN in the window titlebar.

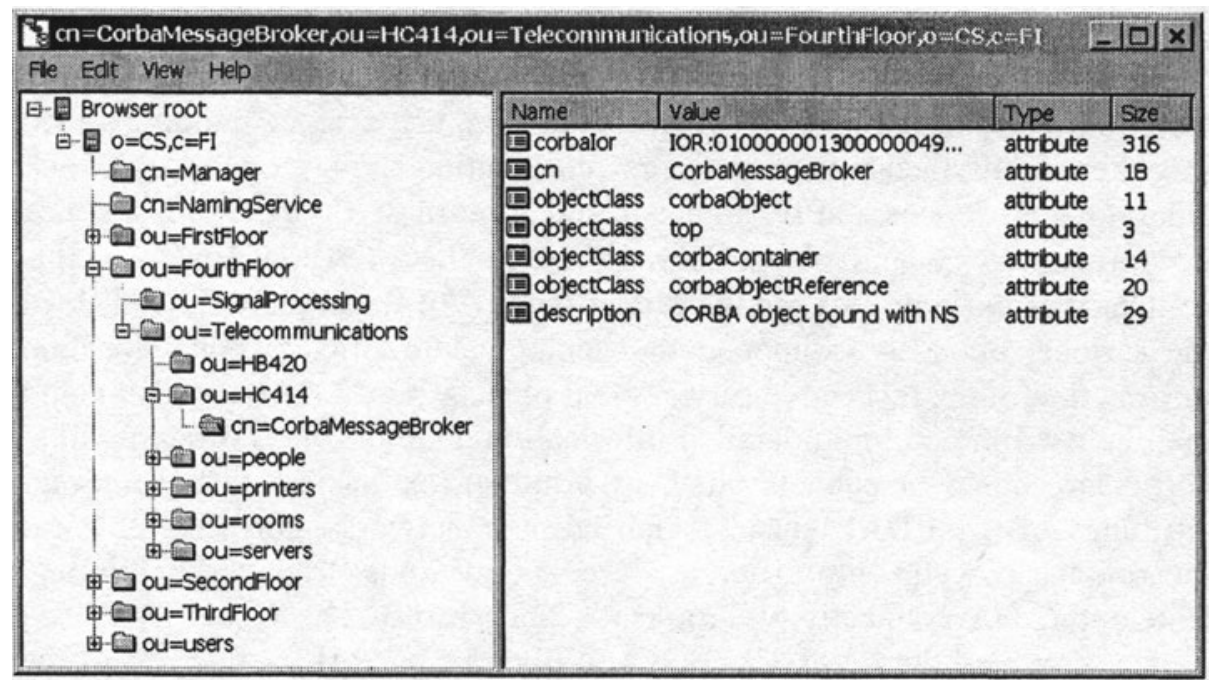

Figure 2. LDAP Browser showing an entry representing a CORBA object

Because the CORBA Naming Service that has been specified by the Object Management Group directly supports the notion of name-to-object associations using name bindings relative to a naming context [6], an entire Naming Service is conceptually a naming graph having hierarchical relationships among parent and child nodes representing contexts and directed edges forming names. This relationship is directly supported by the hierarchical nature of an LDAP directory structure. Also, a name is comprised of a concatenation of components, where components each consist of an id attribute and a kind attribute, with the last component 
denoting the bound object. The Naming Service specifications do not restrict the way in which the naming system should interpret, assign or manage these attributes. Thus our prototype maps these structures to be directly relevant to the entries in the LDAP tree, in such a way that the kind attribute is mapped to the corresponding LDAP entry's attribute type, such as "cn" or "ou", and the $i d$ attribute is mapped to its value. Therefore, if the CORBA Naming Service binds itself to the LDAP Tree with the DN of " $\mathrm{o}=\mathrm{CS}, \mathrm{c}=\mathrm{FI}$ ", then

"ou=FourthFloor,ou=Telecommunications, ou=HC414,cn=CorbaMessageBro ker" would actually be a valid name string for binding or resolving an object using our prototype Naming Service, as it would convert the given name sequence to a string representing the DN of the LDAP entry.

At the moment, the Naming Service implements four methods: bind, unbind and rebind are used by CORBA server objects to register themselves, and resolve is used by CORBA client objects to find the IORs of server objects corresponding to their names from the Naming Service.

\subsection{Example usage scenario}

For starting the Naming Service, the following steps are undertaken:

1. OpenLDAP server daemon slapd, must first be running.

2. The OpenSLP server daemon, slpd is launched on all machines which desire to host SAs.

3. The CORBA Naming Service application is launched, and binds to a well known location to the LDAP back-end. This will be known as the root context of the Naming Service. At the moment, this is configured statically. However this can be easily extended to be dynamic as well.

4. The Naming Service registers itself to its local slpd, passing its URL (e.g. "service:namingservice://myhost.company.com")and its stringified IOR as an attribute.

5. The Naming Service is now ready to serve CORBA objects.

Registering a CORBA server application with the Naming Service would proceed in the following manner:

1. CORBA Server starts up, and behaves as a UA which multicasts a Service Request packet to the Administratively Scoped SLP Multicast Address [17], 239.255.255.253, requesting for a service type "namingservice". Currently, UAs use default scopes with a multicast packet TTL value of 8 .

2. The SA responsible for the Naming Service unicasts a Service Reply back to the requesting UA, returning its URL and thus specifying the location of the Naming Service. 
3. Armed with the URL, the UA once again multicasts an Attribute Request packet requesting for the IOR attribute for that URL.

4. The SA once again unicasts directly to the UA with an Attribute Reply, furnishing it with the IOR attribute registered with that URL.

5. Armed with enough knowledge now, the CORBA Server application contacts the Naming Service directly with the IOR it possesses, and registers itself with the Naming Service using the bind call with 2 parameters: its name and its IOR.

6. The Naming Service maps the information into an LDAP call and stores the information in its LDAP database backend.

In order for a CORBA client finding a CORBA server, it does the following:

1. The CORBA client first discovers the Naming Service in a similar way to the CORBA Server.

2. The Client calls the resolve call of the Naming Service, passing the name of the CORBA server object as a parameter and obtaining an IOR from the Naming Service with which the client then invokes methods on the CORBA server object.

\section{RESULTS}

The basic network topology used for developing and testing the prototype implementation is depicted in Figure 3. All 3 subnets were multicast aware, with the FreeBSD machine hosting the LDAP, Naming Service, CORBA server objects and SLP Service Agents.

As an initial indication of the performance of the prototype, the timing measurements that were recorded are tabulated in Table 1. All SLP and CORBA client calls in Table 1 were made from the SunBlade machine to the FreeBSD machine. For the Naming Service, the time taken to execute a single resolve call was measured. The time taken to execute the same call was also measured against MICO's native naming service, which runs as a standalone daemon in the network. For SLP measurements we timed the discovery of the Naming Service. No DAs were employed in this topology, hence communication occurred directly between the UA in the SunBlade machine and the SA in the FreeBSD machine. The measurements were taken over a period of approximately 3 days. 


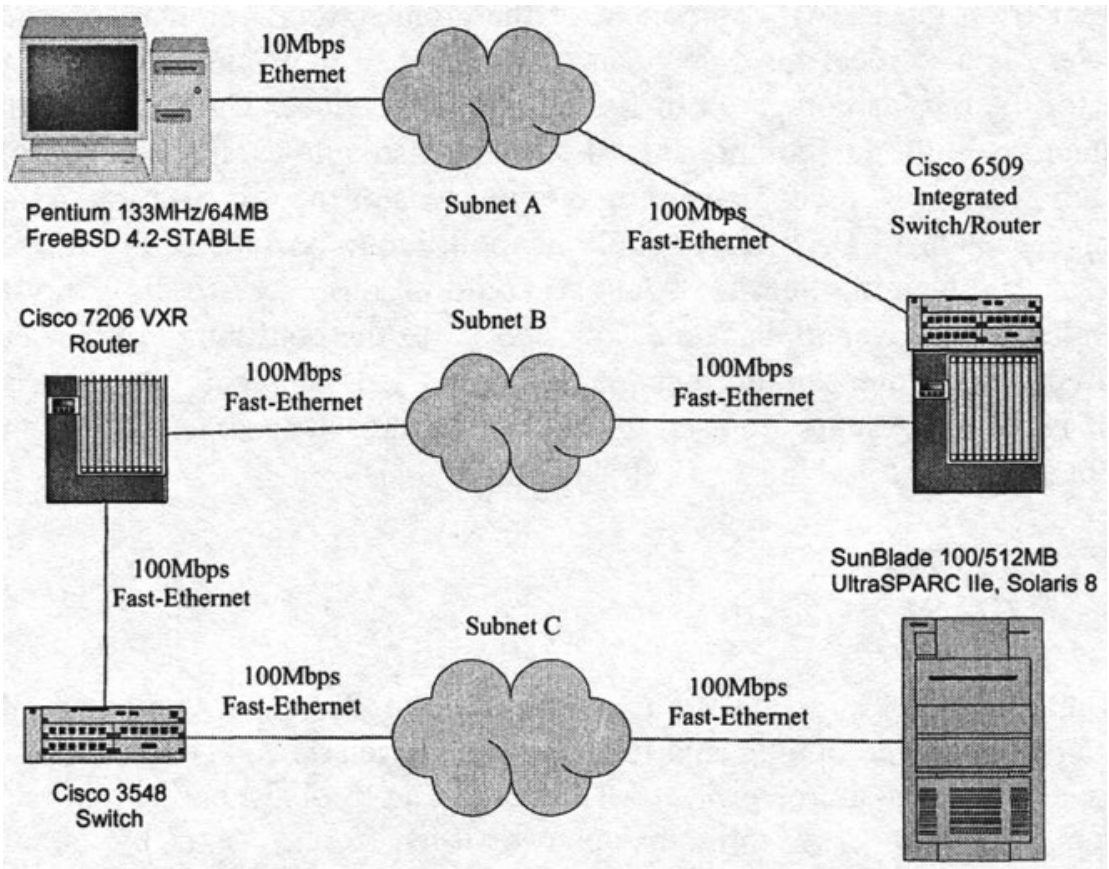

Figure 3. Physical Network Topology

Approximately two-thirds of the execution time of the resolve call was consumed by the LDAP operation, while a small fraction was consumed in the Naming Service front-end that serves as a gateway by mapping parameters and return results to and from the CORBA Naming Service API and the OpenLDAP API. On the other hand, to use MICO's own Naming Service, it is necessary to initially run an object adapter daemon called micod, create an entry for the naming service in an implementation repository and pass the clients the address of the naming service. A fair bit of manual intervention, static configuration and using commandline options are hence necessary. Also, the entries of the Naming Service are stored in memory.

Table 1. Timing measurements in milliseconds

\begin{tabular}{lccc}
\hline & Prototype & MICO's Naming Service & SLP \\
\hline Average duration & 36.5843 & 3.60773 & 4.24144 \\
Maximum duration & 148.506 & 34.252 & 10.203 \\
Minimum duration & 34.289 & 3.213 & 3.960 \\
\hline
\end{tabular}

Thus, bearing in mind the objectives laid out in Section 3, we firmly believe that the offset in performance of our prototype implementation is justified by virtue of the scalable, replicating and fault-tolerant properties 
inherent from the LDAP component of the architecture. Automatic service discovery is also ideal for object services, owing to their more volatile and migratory nature as compared to fixed network services. The design of the architecture with the components described also allows for the flexible interaction amongst them, depending on the needs of the organization. As an example, the OpenLDAP server could be modified to become SLP-aware so that even the Naming Service front-end could perform service discovery to dynamically discover its backend, as opposed to the configuration that our prototype used. Interworking between SLP and LDAP is being studied, so that if Directory Agents are used in the architecture, SLP service URLs and attributes can be stored in an LDAP Directory. [10]

\section{CONCLUSIONS}

Almost all the software components used in designing, building and testing the prototype implementation have been based on code obtained from highly active open-source projects. This has made troubleshooting and bugsolving relatively easy through open mail-list forums and by simply browsing through the code. Minor problems, such as UAs in OpenSLP insisting on multicasting to search for DAs even when configured not to, were quickly solved, and more optimal service discovery times were achieved (from a very rough estimate, UAs using Solaris's SLP library for service discovery took far longer, about 12 seconds).

At the moment, SLP over IPv6 within our prototype has yet to be tested. However, the following are changes required to have the Service Location Protocol work over IPv6. These changes include [11]:

- Eliminating support for broadcast SLP requests

- Address Specification for IPv6 Addresses in URLs

- Use of IPv6 multicast addresses and IPv6 address scopes

- Restricted Propagation of Service Advertisements

The architecture proposed in this paper also does not preclude the use of any security models and can remain fully conformant to any and all security mechanisms standardized by the many RFCs and other specification documents for its various components. As an example, SLP authentication and LDAP security and access control mechanisms can be used to enhance the architecture to allow employees and regular users to use all standard services of the organization's internal network, but restricting visitors and anonymous users to a smaller subset, perhaps by presenting a different location service front-end during service discovery which has a more limited level of access and visibility of the LDAP directory. 
Being able to store, access and manipulate data stored in a common directory format with LDAP also has a huge advantage in being able to use common LDAP browsers (with varying levels of quality) supported in many platforms. This allows a far greater ease of management and maintenance than having to maintain several different service models, each having their own customized administration tools. This also significantly reduces the learning curve for efficient tool use.

The network computing industry has eagerly embraced technologies, welcoming an ever-increasing variety of new service discovery protocols and object architectures. With this abundance now offered across a wide collection of environments, technologies that offer standardized interfaces for the discovery process, while supporting communication for several different types of service access technologies, will provide the greatest achievable interoperability and resilience in the long-term. In this respect, the proposed architecture in this paper holds good promise for supporting enterprise-level next-generation computing needs.

\section{REFERENCES}

[1] Sun Microsystems: Jini Network Technology, http://www.sun.com/jini/

[2] OMG: The Common Object Request Broker: Architecture and Specification. CORBA V2.3, June 1999.

[3] Microsoft Corporation: .NET http://msdn.microsoft.com/net

[4] IETF: RFC 2608, "Service Location Protocol, Version 2", June 1999.

[5] Microsoft Corporation: Universal Plug and Play forum, http://www.upnp.org

[6] OMG: Naming Service Specification, February 2001.

[7] IETF: RFC 2251, "Lightweight Directory Access Protocol (v3)", Dec 1997.

[8] Erik Guttman, James Kempf: Automatic Discovery of Thin Servers: SLP, Jini and the SLP-Jini Bridge, IECON, San Jose, 1999.

[9] Apple Computer Inc.: Mac OS X Server - Network \& Security http://www.apple.com/macosx/server/networksecurity.html

[10]IETF: RFC 2609, "Service Templates and Service: Schemes", June 1999.

[11]IETF: RFC 3111, "Service Location Protocol Modifications for IPv6", May 2001.

[12] The OpenSLP Project, http://www.openslp.org

[13] The OpenLDAP Project, http://www.openldap.org

[14]MICO - Mico Is COrba, http://www.mico.org 
[15]IETF: RFC 2714, " Schema for Representing CORBA Object References in an LDAP Directory", October 1999.

[16] Softerra LDAP Browser, http://www.ldapadministrator.com

[17] IETF: RFC 2365, "Administratively Scoped IP Multicast", July 1998. 